\title{
NOTES
}

\section{Inclusion of Polyaniline in the Channel Constructed from Tris(2,3-naphthylenedioxy)cyclotriphosphazene}

\author{
Isao YAMAGUCHI $^{\dagger}$ and Takakazu YAMAMOTO ${ }^{\dagger}$ \\ Chemical Resources Laboratory, Tokyo Institute of Technology, \\ 4259 Nagatsuta, Midori-ku, Yokohama 226-8503, Japan
}

(Received September 29, 2005; Accepted January 12, 2006; Published May 15, 2006)

\begin{abstract}
KEY WORDS Polyaniline / Cyclotriphosphazene / Inclusion Adduct /
\end{abstract}
[doi:10.1295/polymj.38.495]

Inclusion adducts of conducting polymer which form nano-ordered structures have attracted strong interest due to their potential application to a molecular wire. Cyclodextrin and zeolite were used as host materials to include conducting polymers such as polyaniline,${ }^{1-3}$ polypyridine, ${ }^{4}$ and polythiophene. ${ }^{5-8}$

Cyclotriphosphazenes (CP's) often form a selfassembled structure with channels which can include various organic guest molecules, especially when they are crystallized in the presence of guest organic molecules. ${ }^{9}$ For example, NapCP (tris(2,3-naphthylenedioxy)cyclotriphosphazene) forms the channel structure illustrated below. Size of the channel can be controlled by the side aromatic substituents; tris(1,2-phenylenedioxy)cyclotriphosphazene, $\mathrm{PhCP}$, and NapCP give the channels with about 5 and $10 \AA$ hole diameters, respectively. ${ }^{9}$ These CP's recognize the size of the guest molecule to form the inclusion adduct, and are applied to separation of isomers ${ }^{10}$ and to isolation of single polymer chain. ${ }^{11,12}$ For example, Allcock reported stereocontrolled polymerization of diene compounds included in the channel consisted of PhCP. ${ }^{11}$
Recently, isolation of single poly(ethylene oxide), PEO, chain was achieved by incorporation in the channel. ${ }^{13}$

Investigation of chemical and physical properties of conducting polymers included in the channel of CP's will provide fundamental information about the conducting polymer. However, there has been no report on inclusion of conducting polymer in the channel of CP's to our knowledge. Herein we report preparation of an inclusion adduct of emeraldine base form of polyaniline (PANI) with NapCP and thermal, optical and electrical properties of the adduct.

PANI used in this study was synthesized according to the literature ${ }^{14}$ and had a number-average molecular weight $\left(M_{\mathrm{n}}\right)$ and weight-average molecular weight $\left(M_{\mathrm{w}}\right)$ of $6.9 \times 10^{3}$ and $28.3 \times 10^{3}$, as determined by GPC. Standing an $N$-methyl-2-pyrrolidone (NMP) solution of PANI (e.g., $2.5 \times 10^{-2} \mathrm{M}$ monomeric unit) and NapCP (e.g., $\left.2.5 \times 10^{-2} \mathrm{M}\right)$ at $20^{\circ} \mathrm{C}$ for 7 days gave an inclusion adduct, PANI/NapCP, as a deep green precipitate. Although both PANI and NapCP were soluble in NMP, the product was insoluble in

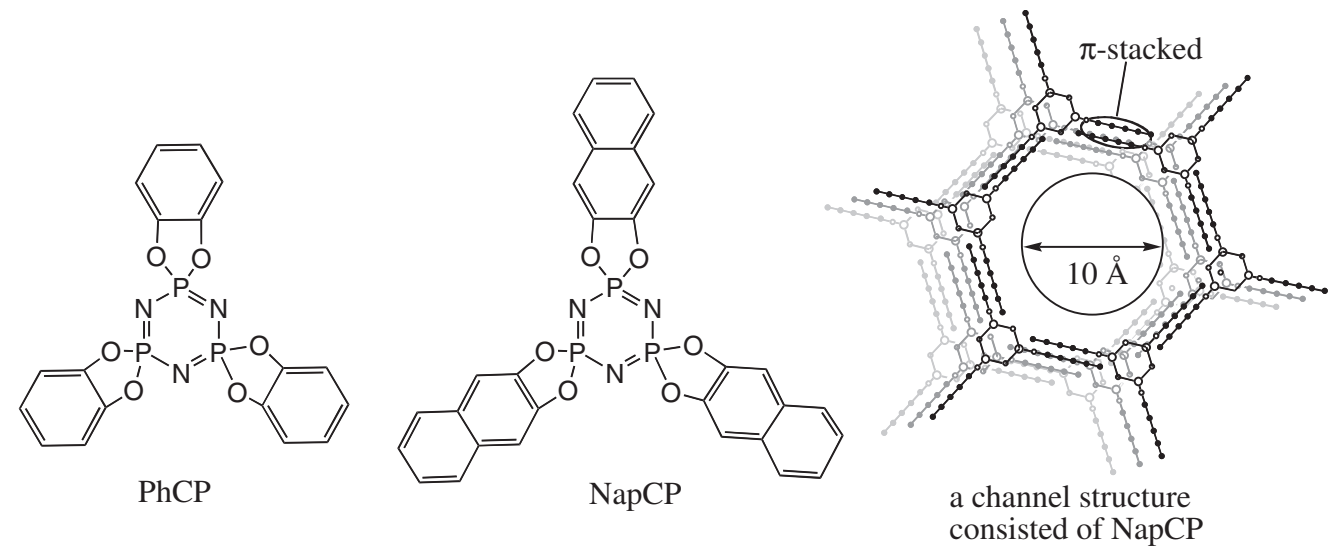

Chart 1. Examples of CP's and molecular assembly of NapCP. CP's form a porous columnar three dimensional structure. ${ }^{9}$

${ }^{\dagger}$ To whom correspondence should be addressed (Tel: +81-45-924-5220, Fax: +81-45-924-5976, E-mail: tyamamot@ res.titech.ac.jp). 


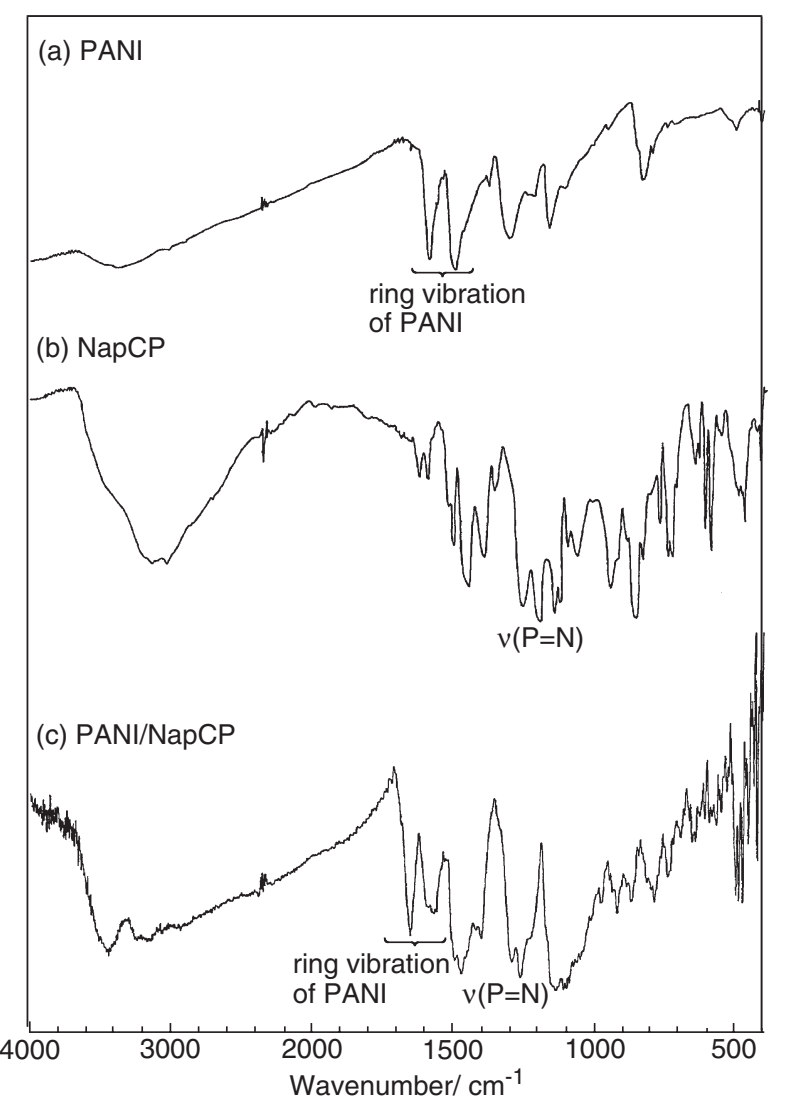

Figure 1. IR spectra of (a) PANI, (b) NapCP, and (c) PANI/ NapCP.

the solvent. It is known that standing a solution containing polyethylene and CP's gives an inclusion adduct and solubility of CP's is altered according to formation of the inclusion adduct. ${ }^{13}$ Repeated washing with NMP removed PANI and NapCP, and the insoluble inclusion product, a PANI/NapCP adduct, was isolated. The $\mathrm{C} / \mathrm{N}$ analytical value of PANI/NapCP (ca. 5.8) was smaller than that of NapCP (ca. 8.6= $(30 \times 12) /(3 \times 14))$, indicating that PANI was incorporated in the channel of NapCP. Formation of the inclusion adduct was also confirmed by IR spectroscopy and thermal analysis as described below, however, content of PANI and NapCP was not able to be determined due to the presence of a naked part of PANI in the inclusion adduct (vide infra).

Figure 1 depicts IR spectra of PANI, NapCP, and the PANI/NapCP adduct. The IR spectrum of the PANI/NapCP adduct shows a $v(\mathrm{P}=\mathrm{N})$ absorption at $1250 \mathrm{~cm}^{-1}$. Ring vibration peaks of PANI in the PANI/NapCP adduct are observed at a higher wavenumber by about $40 \mathrm{~cm}^{-1}$ than those of PANI itself, suggesting a change of the polymer conformation. A similar shift of the ring vibration peaks of PANI to a higher wavenumber was observed in the IR spectrum of PANI included in the crystalline channel of zeolite. ${ }^{1}$ The IR spectrum of the PANI/NapCP adduct

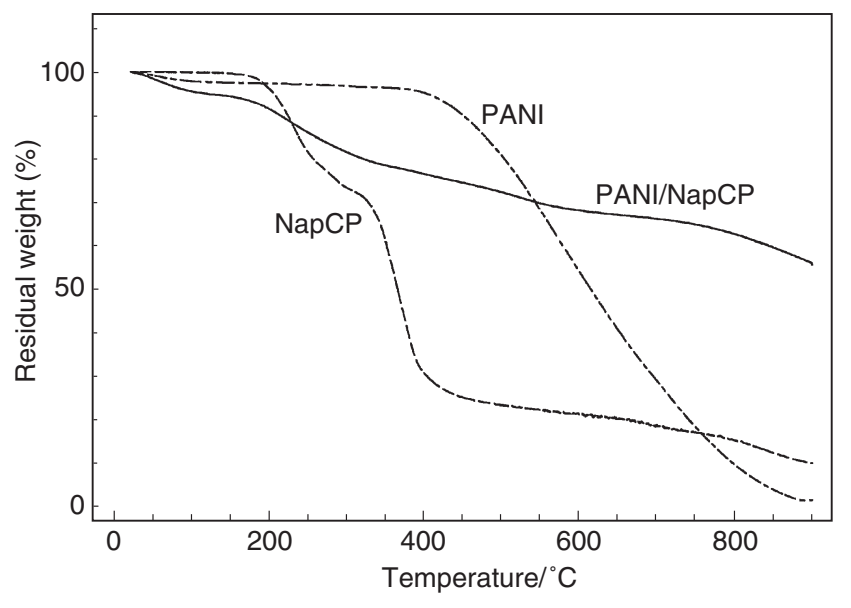

Figure 2. TGA curves of PANI, NapCP, and PANI/NapCP. At a heating speed of $10^{\circ} \mathrm{C} \mathrm{min}^{-1}$ under $\mathrm{N}_{2}$ atmosphere.

was different from that of a mixture of PANI and NapCP which was obtained by simply grinding a mixture of powdery PANI and NapCP in an agate mortar.

TGA data shown in Figure 2 indicate that the PANI/NapCP adduct has high thermal stability and that PANI included in the channel of NapCP possesses a higher thermal stability than PANI itself. PANI and NapCP show $85-100 \%$ weight loss at $900^{\circ} \mathrm{C}$, whereas the PANI/NapCP adduct keeps about $60 \%$ weight at the temperature. It was reported that thermal stability of PANI was improved by inclusion in the channel of the mesoporous aluminosilicate, and the higher stability was attributed to depression of diffusion of PANI in the channel. ${ }^{15}$

The DSC curve of the PANI/NapCP adduct is also considerably different from that of the ground mixture of PANI and NapCP. The DSC curve shows a peak assigned to a melting transition of PANI in the PANI/ NapCP adduct at a somewhat lower temperature $\left(177^{\circ} \mathrm{C}\right)$ than that of the ground mixture of PANI and NapCP $\left(186^{\circ} \mathrm{C}\right)$. It has been reported that thermal properties of $\mathrm{PEO}$ and $\mathrm{PhCP}$ are also changed by the formation of a $\mathrm{PEO} / \mathrm{PhCP}$ inclusion compound and DSC curve of the PEO/PhCP adduct shows no distinct peak assigned to melting of PEO. ${ }^{13}$ Observation of the DSC peak due to the melting transition of PANI for the PANI/NapCP adduct suggests that there may exist free unincluded part of PANI. However, the change of solubility and the improvement of thermal resistance of the PANI/NapCP adduct support the notion that most part of PANI is included in the NapCP channel.

PANI included in the crystalline channel of zeolite showed poor solubility, however, a suspension of the PANI/zeolite inclusion adduct in dimethyl sulfoxide (DMSO) was reported to be susceptible to UV-vis measurements, and the adduct showed a peak of PANI at $400 \mathrm{~nm} .{ }^{1}$ Although the PANI/NapCP adduct was 


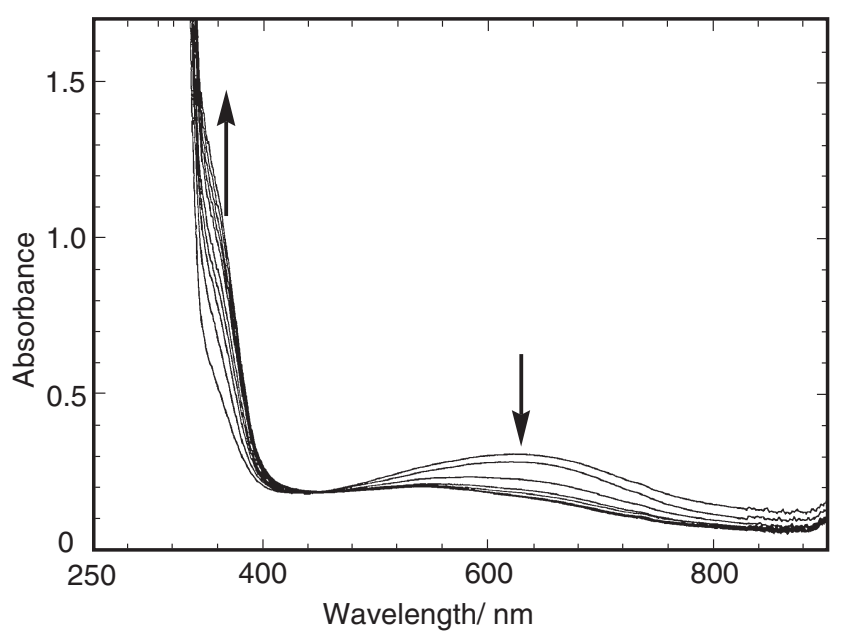

Figure 3. Changes of UV-vis spectrum of a DMSO suspension of the PANI/NapCP adduct during reduction with $\mathrm{N}_{2} \mathrm{H}_{4}$ at $25^{\circ} \mathrm{C} . \mathrm{N}_{2} \mathrm{H}_{4} \cdot \mathrm{H}_{2} \mathrm{O}$ was added to the suspension. $\left[\mathrm{N}_{2} \mathrm{H}_{4}\right]=1 \times$ $10^{-2} \mathrm{M}$. Time $=0,600,1200,1800,2400,3000,3600,4200$, $4800,5400 \mathrm{~s}$ (final state).

slightly soluble in DMSO, measurement of its UV-vis spectrum in a suspension in DMSO was also possible, and reduction of PANI in the PANI/NapCP adduct with $\mathrm{N}_{2} \mathrm{H}_{4}$ was followed by $\mathrm{UV}$-vis spectroscopy. Figure 3 shows changes of the UV-vis spectrum of PANI/NapCP during the reduction with $\mathrm{N}_{2} \mathrm{H}_{4}$ at $25^{\circ} \mathrm{C}$. Both emeraldine base polyaniline (PANI) and its reduced form (leucoemeraldine base, L-PANI) give UV-vis absorption band at $360 \mathrm{~nm}$, whereas only PANI shows the UV-vis peak at $640 \mathrm{~nm}$ assigned to the $\pi-\pi^{*}$ transition of quinoid rings in PANI. The peak at around $640 \mathrm{~nm}$ decreased with time due to reduction of PANI to L-PANI. The reduction reaction followed the pseudo-first-order kinetics in the presence of an excess amount of $\mathrm{N}_{2} \mathrm{H}_{4}$. The pseudo-firstorder rate constant $k$ is $9.2 \times 10^{-4} \mathrm{~s}^{-1}$, whose value is larger than that $\left(k=4.0 \times 10^{-4} \mathrm{~s}^{-1}\right)$ of reduction reaction of PANI at the same concentration of $\mathrm{N}_{2} \mathrm{H}_{4}$ $\left(1.0 \times 10^{-2} \mathrm{M}\right)$. Occurrence of the reduction of original PANI in the PANI/NapCP adduct suggests that proton and electron can move even in PANI included in the channel.

Iodine doping of the PANI/NapCP adduct raised its electronic conductivity by three orders of magnitude to give a semiconducting material with an electrical conductivity of $5 \times 10^{-4} \mathrm{~S} \mathrm{~cm}^{-1}$. The electrical conductivity of the iodine-doped PANI/NapCP adduct is comparable to that of iodine-doped PANI ( $\sigma=$ $10^{-3} \mathrm{~S} \mathrm{~cm}^{-116}$ ). It was reported that PANI fully included by $\beta$-cyclodextrins did not receive the doping with $\mathrm{I}_{2}{ }^{3}$

In conclusion, a new inclusion adduct of PANI with NapCP was obtained. Thermal stability of PANI was improved by the inclusion in the channel constructed from NapCP. The quinoid rings in PANI in the inclusion adduct received reduction with $\mathrm{N}_{2} \mathrm{H}_{4}$. PANI in the inclusion adduct accepted $\mathrm{I}_{2}$-doping and was converted into a semiconducting material.

Acknowledgment. This research was partially supported by the Ministry of Education, Culture, Sports, Science and Technology, Grant-in-Aid for Young Scientists (B), 14750704, 2002.

\section{REFERENCES}

1. P. Enzel and T. Bein, J. Phys. Chem., 93, 6270 (1989).

2. C.-G. Wu and T. Bein, Science, 264, 1757 (1994).

3. T. Shimomura, T. Akai, T. Abe, and K. Ito, J. Chem. Phys. 116, 1753 (2002).

4. I. Yamaguchi, N. Ismayil, and T. Yamamoto, Kobunshi Ronbunshu, 57, 472 (2000).

5. P. Enzel and T. Bein, J. Chem. Soc., Chem. Commun., 1326 (1989).

6. C. Lagrost, K. I. C. Ching, J.-C. Lacroix, S. Aeiyach, M. Jouini, P.-C. Lacaze, and J. Tanguy, J. Mater. Chem., 9, 2351 (1999).

7. L. A. Siegel and J. H. van den Hende, J. Chem. Soc. A, 817 (1967).

8. I. Yamaguchi, K. Kashiwagi, and T. Yamamoto, Macromol. Rapid Commun., 25, 1163 (2004).

9. H. R. Allcock and M. T. Stein, J. Am. Chem. Soc., 96, 49 (1974).

10. H. R. Allcock and N. J. Sunderland, Macromolecules, 34, 3069 (2001).

11. H. R. Allcock, G. K. Dudley, and E. N. Silverberg, Macromolecules, 27, 1039 (1994).

12. H. R. Allcock, E. N. Silverberg, G. K. Dudley, and S. R. Pucher, Macromolecules, 27, 7550 (1994).

13. A. P. Primrose, M. Parvez, and H. R. Allcock, Macromolecules, 30, 670 (1997).

14. M. Abe, A. Ohtani, Y. Umemoto, S. Akizuki, M. Ezoe, H. Higuchi, K. Nakamoto, A. Okuno, and Y. Noda, J. Chem. Soc., Chem. Commun., 1736 (1989).

15. C.-G. Wu and T. Bein, Science, 264, 1757 (1994).

16. H. K. Chaudhari and D. S. Kelkar, Polym. Int., 42, 380 (1997). 